ability of patients with rheumatoid arthritis to respond to preformed crystals using both in vivo and in vitro techniques.

Needle-shaped MSU crystals 2-10 $\mu \mathrm{m}$ long were prepared by modification of the method of Seegmiller et al. ${ }^{2}$; chemical purity was checked by infrared spectrophotometry. Crystals were suspended in sterile saline (skin testing and C3 activation) or Eagle's medium (polymorphonuclear (PMN) interaction) and briefly sonicated before use to reduce aggregation.

(1) Intradermal skin testing. The diameter of erythema produced by intradermal injection of MSU crystals ( $5 \mathrm{mg}$ in $0.2 \mathrm{ml}$ suspension) was measured by a single observer as previously described. ${ }^{3}$ Fifteen patients with seropositive rhe umatoid arthritis (mean age $49 \cdot 1$ years, mean DAT $1: 640), 15$ patients with osteoarthritis (mean age 63.8 years), and 12 normal controls (mean age 26.2 years) showed no significant difference in mean forearm erythema 24 and 48 hours after injection (patients with rheumatoid arthritis: $29 \cdot 8 \pm 6 \cdot 2 \mathrm{~mm}$ after $24,27 \cdot 8 \pm 7 \cdot 3$ after 48 ; patients with osteoarthritis: $32.4 \pm 4.4$ and
$14 \cdot 7 \pm 8 \cdot 1 ;$ and controls $12 \cdot 2 \pm 6$ $10 \cdot 8 \pm 5 \cdot 1)$.

(2) Polymorph phagocytosis and enzyme release. PMNs from patients with rheumatoid arthritis $(n=10)$ and controls $(n=10)$ exposed to MSU crystals (1\%) for 1 hour showed no significant difference in cell attachment/phagocytosis, enzyme release or viability.

(3) Complement activation. Serum C 3 activation produced by 30 minutes' incubation with MSU crystals (1\%) was quantified by densitometry after electrophoretic separation and immunofixation as previously described. ${ }^{4}$ No difference was observed between 10 patients with rheumatoid arthritis (mean DAT $1: 640$ ) and 10 normal sera in the $\%$ of C3 split in excess of saline control $(37.8 \pm 4.9 \%$ and $36 \cdot 7 \pm 3.2 \%$ respectively). C3 activation via the alternative pathway-demonstrated by incubation with EGTA ( $8 \mathrm{mmol})$ and $\operatorname{MgCl}_{2}(0.3 \mathrm{mmol})$-was also similar $(21 \cdot 1 \pm 4 \cdot 2 \%$ and $18.5 \pm$ $3 \cdot 1 \%$ respectively).

These results show that patients with seropositive rheumatoid arthritis mount normal in vitro and in vivo inflammatory responses to MSU crystals. We therefore discount previous suggestions that the negative correlation between rheumatoid arthritis and gout results from a difference in host response. A more plausible explanation is that the connective tissues of patients with rhe umatoid arthritis are not conducive to MSU crystal formation.

We would like to acknowledge the financial support of the Arthritis and Rheumatism Council.

\section{References}

1 Wallace D J, Klinenberg J R, Morhairn D, Berlanstein B, Biren P C, Callis G. Coexistent gout and rheumatoid arthritis: case report and literature review. Arthritis Rheum 1979; 22: 81-6.

2 Seegmiller J E, Howell D R, Malawista $S$ E. The inflammatory reaction to sodium urate. JAMA 1962; 180: 469-77.

3 Dieppe P A, Doherty M, Papadimitriou G M. Inflammatory responses to intradermal crystals in healthy volunteers and patients with rheumatic diseases. Rheumatology International 1982; 2: 55-8.

4 Doherty M, Whicher J T, Dieppe P A. Activation of the alternative pathway of complement by monosodium urate monohydrate crystals and other inflammatory particles. Ann Rheum Dis (in press).

\title{
Ultrastructural observations of crystals in articular cartilage of aged human hip joints
}

\author{
IRENIA MARANTE, R. MACDOUGALL, A. ROSS, AND R. A. STOCKWELL \\ From the Department of Anatomy, University Medical School, Edinburgh EH8 $9 A G$
}

The occurrence of calcification in the normally uncalcified zones of human articular cartilage is well documented ${ }^{1}$ and crystal morphology at the ultrastructural level in osteoarthritic cartilage has been described by earlier workers. ${ }^{2}$ We showed the presence of crystals, probably apatite, in macroscopically normal articular cartilage of the aged human hip joint.

Femoral heads were obtained from eight women undergoing operation for hip arthroplasty after subcapital fracture. Ages ranged from 65 to 91 years and in four cases fracture of the femoral neck had occurred the day before operation. The cartilage of the whole femoral head appeared smooth, though in several specimens a small area about 3-4 $\mathrm{mm}$ diameter beneath the fovea showed minimal fibrillation. Tissue blocks of smooth cartilage from the superior and inferior surface of the femoral head were fixed in glutaraldehyde, stained with osmic acid, and embedded in araldite. Thin sections were stained with uranyl ace tate and lead citrate and examined by transmission electron microscopy. Unstained sections of non-osmicated tissue fixed in unbuffered $10 \%$ formaline were also examined.
Crystals were found in all specimens, occurring singly or occasionally in clusters. They were mostly $0.2 \mu \mathrm{m}$ in diameter but varied from about 0.05 to $15 \mu \mathrm{m}$. They were often polygonal or rounded in shape but many had a sharply angled square contour and were considered to be cuboidal. Several crystals were enclosed by an electron dense lamina, possibly a membrane, adherent to the surrounding cartilage matrix. Many 'empty' areas lined by a similar dense lamina were observed; these corresponded in size and shape to the crystals. This may have been due to 
loss of crystals occurring either during tissue preparation or in vivo. Crystals were situated either randomly in the matrix, many of them within a few micrometers of the articular surface, or around the cells. Although the number of crystals varied, this did not seem to be related to the time interval between the fracture and the sampling of the tissue.

$X$-ray emission microanalyses of crystals $(\mathrm{n}=10)$ in formalin fixed sections showed calcium and phosphorus. The ratio of calcium to phosphorus, uncorrected for absorption, fluorescence, and atomic weight was 1.95 (SD 0.30), suggestive of apatite rather than pyrophosphate deposits.
Ultrastructurally articular surfaces were often roughened, though smooth to the naked eye, and showed infiltration with electron dense material. They were only rarely fissured. In many specimens the superficial collagen fibrils retained the normal density of packing and orientation tangential to the surface. More deeply, the fibres could show some disorganisation. Flattened chrondrocytes were often present in the superficial zone. Chrondrocytes in deeper tissue contained irregular lobed nuclei and several lipid droplets in addition to active Golgi and endoplasmic reticulum.

Crystal deposition in the hip joints of these women may be related to osteoporosis, and this requires further investigation. Localisation of the crystals within the cartilage (superficial zone and pericellular regions) corresponds to sites of extracellular lipid in aging articular cartilage generally. Perhaps the most noteworthy features are the occurrence of crystals in non-arthritic hip joints, and their occurrence in all the elderly women in this group.

\section{References}

1 Dieppe $\mathrm{P}$ A. New knowledge of chrondrocalcinosis. J Clin Path 1978; 31, suppl 12: 214-22.

2 Ali S Y. In: Ascenzi E, Bonucci E, de Bernard B, eds. Matrix vesicles. Milan: Wichtig, 1981: 241-7.

\title{
Ultrastructural studies of pyrophosphate crystal deposition in articular cartilage
}

\author{
S. Y. ALI ${ }^{1} S$. GRIFFITHS,${ }^{1} M$. T. BAYLISS,${ }^{1}$ AND P. A. DIEPPE ${ }^{2}$ \\ From the ${ }^{1}$ Institute of Orthopaedics, Royal National Orthopaedic Hospital, Stanmore, Middlesex, and ${ }^{2}$ Bristol Royal \\ Infirmary, Bristol
}

The mechanism of calcium pyrophosphate crystal formation in the articular cartilage of patients suffering from this crystal deposition disease has not yet been fully elucidated. It has been suggested that the earliest pyrophosphate crystal deposition is adjacent to chondrocyte lacunae ${ }^{1}$ and has been supported by light microscopical studies. Ultrastructural studies have also been performed on cartilage from patients with chondrocalcinosis but they have been restricted to the study of the changes in the matrix. ${ }^{23}$ We have embarked on an electron microscopical study of articular cartilage specimens from patients with calcium pyrophosphate crystal deposition disease and have combined it with electron probe analysis of the crystals to determine their chemical composition.

By using modified staining techniques it is now possible to show crystals in ultrathin sections of cartilage. Large islands of crystals were seen in the matrix, confirming earlier

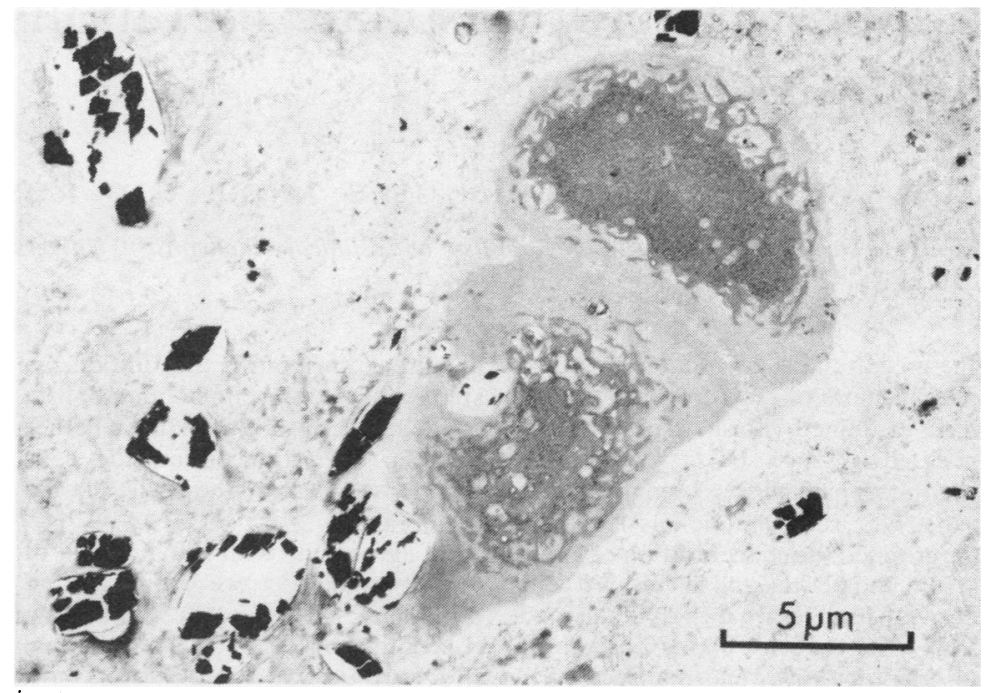

Fig. 1 Electron microscopical picture of two chondrocytes in the matrix of articular cartilage specimen obtained from the knee of a patient with calcium pyrophosphate crystal deposition disease ( 70 year old woman). Note that some of the crystals are adjacent to the chondrocyte and are present within the lacuna. Both the cells have an abnormal amount of glycogen in the cytoplasm. The appearance of the matrix is also abnormal. $\times \overline{5} \overline{5} \tilde{0} \dot{0}$ (original magnification). 